\title{
The Decline of Comprehensiveness and the Path to Restoring It
}

\author{
Timothy J. Judson, MD, MPH ${ }^{7}$, Matthew J. Press, MD, , and Allan S. Detsky, MD, PhD, \\ $C M^{4,5}$
}

'Department of Medicine, University of California, San Francisco, San Francisco, CA, USA; ${ }^{2}$ Primary Care Service Line, University of Pennsylvania Health System, Philadelphia, USA; ${ }^{3}$ Division of General Internal Medicine, Department of Medicine, Perelman School of Medicine, University of Pennsylvania, Philadelphia, USA; ${ }^{4}$ Institute of Health Policy, Management and Evaluation and Department of Medicine, University of Toronto, Toronto, Canada; ${ }^{5}$ Departments of Medicine, Mount Sinai Hospital and University Health Network, Toronto, ON, Canada.

J Gen Intern Med 35(5):1582-3

DOI: $10.1007 / \mathrm{s} 11606-019-05357-0$

(c) Society of General Internal Medicine 2019

A few years ago, a physician was rushing to the airport for a trip to Chicago when he felt his heart begun to race. Fortunately, it resolved within seconds. When he returned home, he saw his primary care physician at a major academic medical center. She recommended an electrocardiogram (ECG), and he asked if he should change into a gown. "Not here," she said. He was instructed to walk to the cardiology offices in a separate building, where he then was told to schedule an appointment for an ECG-the next day. Once the ECG was performed and read by a cardiologist, the result was sent back to the primary care doctor and - three days after the initial encounter-relayed to the patient. The process of evaluating his transient tachycardia contrasted sharply with his experience booking that trip to Chicago. In just a few minutes, on one website, he reserved his flight, hotel, and rental car. These "all-in-one" - or comprehensive-consumer experiences are the norm in many service industries, but are increasingly rare in healthcare.

Comprehensiveness refers to the depth, breadth, and scope of expertise and services provided to a consumer by a specific physician or practitioner. In healthcare, more comprehensive care is associated with improved patient satisfaction, lower costs, and higher quality. ${ }^{1,2}$ In one study, for example, patients cared for by family physicians who billed for a broader range of services had lower costs and fewer hospitalizations. ${ }^{1}$ When patients need multiple, separate interactions with the healthcare system, they miss work, have higher out-of-pocket costs, face often-cacophonous recommendations from various providers, and are less likely to complete the recommended plan.

Across a variety of measures, comprehensiveness is waning in healthcare. Today's family physicians are less likely than

Received July 2, 2019

Accepted September 12, 2019

Published online October 24, 2019 their predecessors to provide prenatal, gynecologic, or pediatric care. ${ }^{1}$ The rate of referrals to specialists is increasing, ${ }^{3}$ and the percentage of the physician workforce practicing primary care and "general" specialties (e.g., general ophthalmology or orthopedics) is shrinking. ${ }^{4}$ At the same time, patients are increasingly seeking "one-stop shopping." They want their health concerns addressed in as few encounters as possible without sacrificing quality of care and access to expertise. ${ }^{2}$

\section{DRIVERS OF THE DECLINE IN COMPREHENSIVENESS}

Why is comprehensiveness declining? One reason is increasing medical specialization. Within general internal medicine, more physicians are narrowing their scope to one practice setting (i.e., hospital medicine). Meanwhile, specialists are increasingly subspecializing. In 2015, $65 \%$ of ophthalmology residents went on to subspecialty fellowships, double the rate in $2000 .^{5}$

Several factors are driving increased specialization. First, there is a higher density of specialists per capita in urban compared with rural areas. In these high-density markets, increased specialization may be pursued as a competitive advantage to meet patient expectations. Second, some physicians may prefer to focus more on depth and less on breadth of knowledge. The more we master a skill, the happier we are to perform it. The impact of mastery on physician training choices is not new, but as technology advances and medical care becomes more complex, this trend towards more subspecialization has likely accelerated. Third, physicians may narrow their scope of practice to reduce malpractice risk (e.g., refer all ECGs to cardiology).

For certain types of healthcare-like intensivists in the ICU or high-volume specialists performing surgery—specialization leads to better outcomes. But in our current system of imperfect coordination, increasing sub-specialization can lead to diffusion of responsibility - a phenomenon known as "bystander apathy"- and increased fragmentation of care. ${ }^{6}$

Consolidation also may play a role in the decline of comprehensiveness. Theoretically, consolidation could allow for better communication between providers and better coordination of care for patients. However, when healthcare entities 
consolidate, they often attempt to achieve economies-of-scale by centralizing services, which may inconvenience patients who now have to travel for ancillary tests, rather than complete them at their local physician's office. Consolidation also lowers the barriers and sometimes increases the incentives for physicians to make referrals within the system, which when unnecessary, can increase fragmentation of care.

A third factor driving the decline of comprehensiveness is fee-for-service reimbursement, in which physicians are incentivized to increase patient volume and make and receive more referrals. The simplest way of increasing relative value units in primary care is to see more patients, leaving less time for each encounter. Even billable services (like ECGs) may not be worth performing in a primary care office if the opportunity cost of doing so - the revenue lost from being unable to use that room and staff to begin another encounter - is greater than the billable fee for the test.

\section{RESTORING COMPREHENSIVENESS}

Comprehensiveness does not necessitate that patients forego specialist expertise, that the healthcare system sacrifices economies of scale, or that physicians accept a net decline in their incomes or satisfaction. If used creatively, digitization can help patients get the right level of expertise while preserving comprehensiveness. For example, through the use of electronic consults, primary care physicians can obtain expert opinions without physically sending patients to specialists, thus streamlining communication and patient experience. Innovations on this model such as targeted automatic e-consults ${ }^{7}$ and specialist-designed workflows for chronic diseases can further empower generalists to manage complex cases.

When in-person specialist care is necessary, clinics can be clustered by patient condition such that patients can get coordinated care in one location. For example, a musculoskeletal center can include physiatrists, orthopedists, rheumatologists, pain specialists, and physical therapists. For these centers to effectively deliver comprehensive care, appointments and treatment plans all need to be fully integrated and coordinated. To preserve economies-of-scale while avoiding extra travel for patients, ancillary services can be located in convenient settings, such as in multispecialty clinic buildings. When separate visits are required, walk-in appointments and open-access online scheduling should be available. Since primary care and family medicine physicians traditionally provide more comprehensive care than specialists, Medicare should consider increasing support for these residency training programs. Finally, to address the growing risk of bystander effect, ${ }^{6}$ training programs and professional societies in all specialties should work to cultivate comprehensiveness as a core component of physician professionalism.

Restoring comprehensiveness requires both convincing physicians and healthcare organizations of its importance and supporting it with a payment system that rewards coordinated, patient-centered care. Most primary care physicians want to provide comprehensive care; but they can only do so within the right payment system. Given its potential for cost-savings, comprehensiveness should be a central aim of value-based payment models. Healthcare systems that function as accountable care organizations, for example, are incentivized to avoid unnecessary, fragmented care. These incentives should translate into programs and processes that promote comprehensiveness.

Comprehensiveness may be declining in medicine, but it is not lost. Patients will search for and choose healthcare systems where their health issues can be addressed efficiently and effectively. In a truly competitive market, patient choices would result in the right balance between comprehensiveness and expertise. In healthcare, however, market distortions such as monopolies and asymmetry of information prevent this balance from occurring. Since market forces have failed to produce comprehensiveness, healthcare systems large and small will need to prioritize it in their strategic visions. If we can travel the globe with one-stop shopping, there is no reason the provision of a patient's healthcare should be any less convenient and comprehensive.

Acknowledgments: We thank Robert Wachter, MD, University of California, San Francisco, for his helpful comments on an earlier draft of the manuscript. He did not receive compensation.

Corresponding Author: Timothy J. Judson, MD, MPH; Department of Medicine University of California, San Francisco, 505 Parnassus Avenue, San Francisco, CA 94143, USA (e-mail: timothy.judson@ucsf. edu).

\section{Compliance with Ethical Standards:}

Conflict of Interest: The authors have no conflicts of interest to disclose.

\section{REFERENCES}

1. Bazemore A, Petterson S, Peterson LE, Phillips RL. More Comprehensive Care Among Family Physicians is Associated with Lower Costs and Fewer Hospitalizations. Ann Fam Med. 2015;13:206-13.

2. Pet Lewis CL, Wickstrom GC, Kolar MM, et al. Patient preferences for care by general internists and specialists in the ambulatory setting. $J$ Gen Intern Med. 2000;15(2):75-83.

3. Barnett ML, Song $\mathbf{Z}$, Landon BE. Trends in physician referrals in the United States, 1999-2009. Arch Intern Med. 2012;172:163-70.

4. Barbey C, Sahni N, Kocher R, Chernew ME. Physician workforce trends and their implications for spending growth. Health Affairs Blog. https:// www.healthaffairs.org/do/10.1377/hblog20170728.061252/full/. Published July 28, 2017. Accessed May 28, 2019.

5. Parke DW. In the pursuit of fellowship. American Academy of Ophthalmology. https://www.aao.org/young-ophthalmologists/yo-info/article/inpursuit-of-fellowship. Published May 14, 2015. Accessed May 28, 2019.

6. Rosenbaum L. The Not-My-Problem Problem. $N$ Engl $J$ Med. 2019;380(9):881-885.

7. Wachter RM, Judson TJ, Mourad M. Reimagining Specialty Consultation in the Digital Age: The Potential Role of Targeted Automatic Electronic Consultations. JAMA. Jun 27, 2019; [epub ahead of print].

Publisher's Note Springer Nature remains neutral with regard to jurisdictional claims in published maps and institutional affiliations. 